\title{
RADIATION-DRIVEN SHOCK AND DEBRIS PROPAGATION DOWN A PARTITIONED PIPE
}

\author{
M. D. FURNISH ${ }^{1}$, R. J. LAWRENCE ${ }^{1}$, C. A. HALL ${ }^{1}$, J. R. ASAY1 ${ }^{1}$, D. L. BARKER ${ }^{2}$, \\ G. A. MIZE ${ }^{2}$, E. A. MARSH ${ }^{2}$, and M. A. BERNARD ${ }^{3}$ \\ ${ }^{1}$ Sandia National Laboratories, Albuquerque NM 87185-1168 USA, ${ }^{2}$ Bechtel Nevada Corporation, P.O. Box 98521, \\ Las Vegas, NV 89193-8521, ${ }^{3}$ Ktech Corporation, 2201 Buena Vista SE, Suite 400, Albuquerque, NM 87106-4265
}

\begin{abstract}
Two experiments have been performed to measure the effects of pulsed radiation loads on the front of small tubular structures, using as an energy source the X-ray fluence produced by a Z-pinch at the Sandia National Laboratories Z Facility. The project had two major goals: to establish the feasibility of using the $Z$ machine to study the phenomenology associated with debris generation and propagation down tubular structures with partitions; and to use the resultant experimental data to validate numerical hydrocodes (shock physics codes) so that we have confidence in their use in analyzing these types of situations. Two tubular aluminum structures $(5$ and $10 \mathrm{~cm}$ long and $1 \mathrm{~cm}$ inside diameter) were prepared, with aluminum partitions located at the front, halfway down the pipe, and at the rear. Interferometry (VISARs) provided multiple velocity histories for all of the partitions. In both experiments, the first barrier, which was exposed directly to the $\mathrm{x}$-ray fluence, was launched into the pipe at a velocity of $\sim 2 \mathrm{~km} / \mathrm{s}$, accelerating to give a mean velocity of $\sim 2.6 \mathrm{~km} / \mathrm{s}$. Loss of plate integrity is inferred from the dispersed launch of the second partition at $\sim 1 \mathrm{~km} / \mathrm{s}$. Wall shocks propagating at $4.5 \mathrm{~km} / \mathrm{s}$ were inferred, although strain gage measurements did not succeed. Post-test metallography showed evidence of melting and partial vaporization of the plates, and turbulent mixing with material from the walls. Calculations qualitatively agree with the observed results, but slightly overpredict debris velocity, possibly due to overestimates of total energy fluence. An application for this work is the study of techniques for line-of-sight shock and debris mitigation on high-power pulse-power facilities such as $\mathrm{Z}$ and its follow-on machines.
\end{abstract}

Keywords: Z-pinch, debris, ALEGRA, VISAR

\section{INTRODUCTION}

As more elaborate experiments are conceived to utilize the $\mathrm{Z}$ accelerator [1], NIF, and other pulsed-power facilities, the risk of experiment failure due to an impact of $X$-ray or laser driven debris increases [2]. At the same time, the study of such debris affords the opportunity to learn about X-ray coupling aspects of weapon effects phenomena. Centimeter-sized hohlraums allow target structures large enough to accommodate a wide variety of diagnostics, while the hohlraum temperatures achieved $(150-250 \mathrm{eV})$ provide a useful spectrum of soft $\mathrm{X}$ rays.

In the present study, we instrumented two tubular structures with multiple velocity interferometry systems (VISARs) and with strain gages to measure the response of these structures to the X-ray flux from the Z-pinch. This process included constructing fiber optic and cable connections to the experimental chamber to accommodate 10 channels of VISAR and three strain gages per test.

Both structures were recovered after their respective tests, and one was subjected to detailed metallographic, microprobe and SEM examination. Results of this examination, and the timeresolved VISAR results, together show that model response to the X-ray flux is a complicated process involving melting, vaporization, tube wall excoriating, and material diffusion into the walls - a stark contrast with a simple model of partitions traveling down the tube as flying plates. 


\section{EXPERIMENTAL CONFIGURATION}

A schematic drawing of the overall tube assembly is shown in Fig. 2.1. The three partitions shown are machined by diamond-turning from 1100 alloy aluminum (essentially pure elemental aluminum), and are $1 \mathrm{~mm}$ thick except for the one most distant from the pinch, which is $3 \mathrm{~mm}$ thick. Diameters are $1 \mathrm{~cm}$. The remainder of the tube structure is machined from 6061-T6 aluminum, an alloy whose dynamic response has been extensively studied over the years.

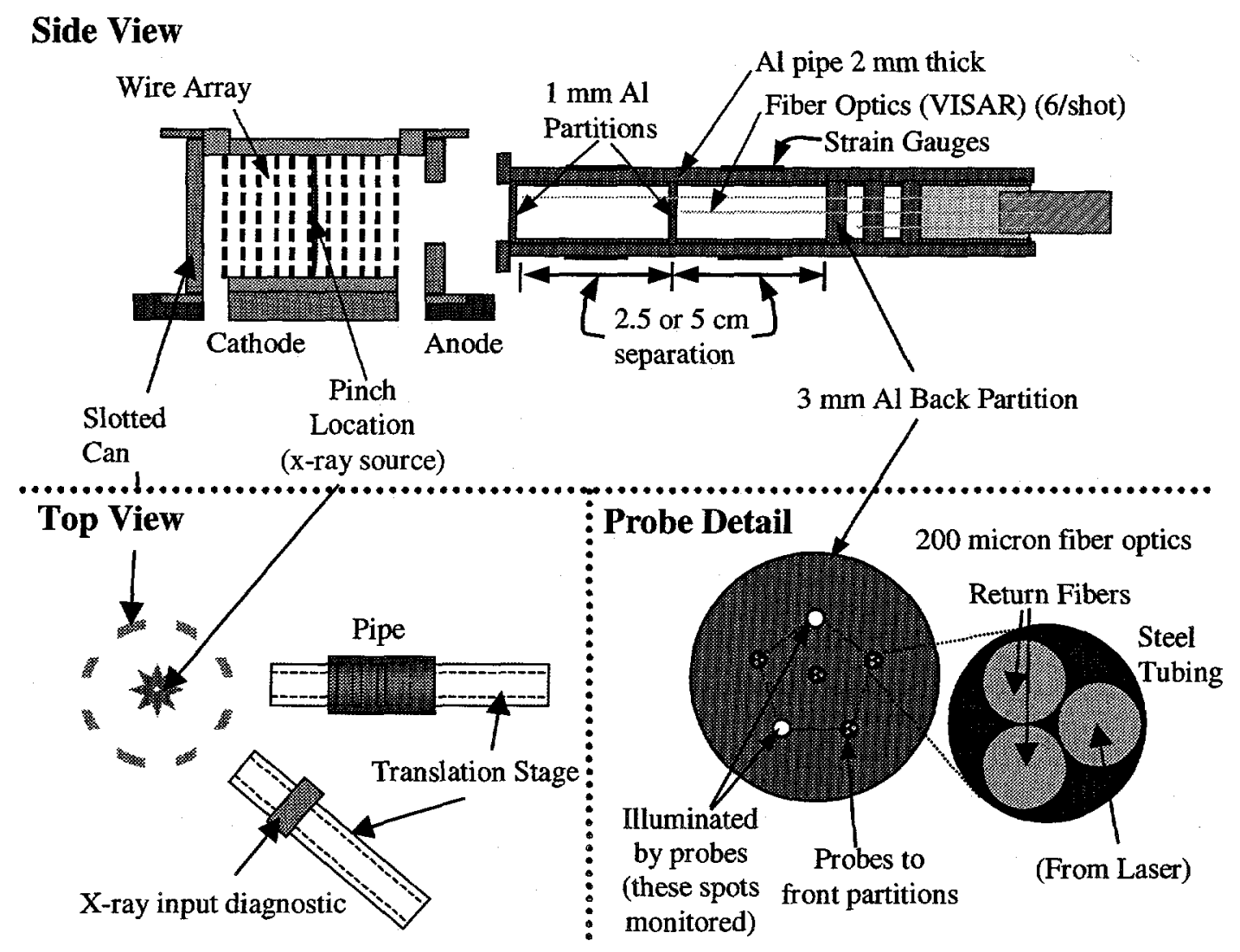

Fig. 2.1. Schematic of tube experiment. Long tube has partitions separated by $5 \mathrm{~cm}$; short tube has $2.5-\mathrm{cm}$ separation.

Lenseless fiber optic probes were introduced prior to emplacement of the partitions, using a jig to ensure that the ends were $0.5 \mathrm{~mm}$ from the final position of the partition. Each of these probes was composed of 3 fiber-optics (one for light from the laser; the other two to transmit doppler-shifted light to the VISARS) in a bundle encased in a $1 \mathrm{~mm}$ diameter steel syringe tube. The $0.5-\mathrm{mm}$ separation has been found to be a value allowing usable intensity return to the VISARs. Smaller spacings result in too little overlap between the area illuminated by the fiber from the laser and the areas contributing to the light returned to the VISARs. The configuration of holes for the fiber optics is shown in Fig. 2.2. Because of the construction of these probes, the motion of only one of the partitions at the center could be monitored.

Strain gages were placed on the outside of the tube wall for both tests (see Fig. 2.1). The requirement for mounting points for associated circuits, as well as for gage protection from the $\mathrm{X}$-ray flux, necessitated the use of a large housing for the tube experiment.

As a control, an aluminum disk similar to the front partition was placed adjacent to a second hohlraum port and monitored by VISAR. This is labeled "X-ray input diagnostic" in Fig. 2.1.

Fluence measurements were performed with diagnostics already in-place at $\mathrm{Z}$ for characterizing source terms. These did not affect the fluence delivered to the tube experiment or 


\section{DISCLAIMER}

This report was prepared as an account of work sponsored by an agency of the United States Government. Neither the United States Government nor any agency thereof, nor any of their employees, make any warranty, express or implied, or assumes any legal liability or responsibility for the accuracy, completeness, or usefulness of any information, apparatus, product, or process disclosed, or represents that its use would not infringe privately owned rights. Reference herein to any specific commercial product, process, or service by trade name, trademark, manufacturer, or otherwise does not necessarily constitute or imply its endorsement, recommendation, or favoring by the United States Government or any agency thereof. The views and opinions of authors expressed herein do not necessarily state or reflect those of the United States Government or any agency thereof. 


\section{DISCLAIMER}

Portions of this document may be illegible in electronic image products. Images are produced from the best available original document. 
the input diagnostic.

To effect the multibeam VISAR diagnostics required for this experiment, a 107-foot bundle of 40 fibers (200-micron multimode low OH FHP, Polymicro) and a 5-foot feedthru were manufactured. These connected the experiment in the evacuated center section of the $Z$ facility with the screen room containing the VISAR and recording systems. They afforded the opportunity for flexibility in future tests.

Two multibeam Valyn International VISAR interferometers were used (one VLNV-03 and one VLNV-04 model) to monitor the motion of the partitions. Each of these interferometers has the capability of measuring velocity histories for up to 7 spots. In these experiments, both interferometers measured the velocity history of two points on the front partition (closest to the pinch; both at $0.25-\mathrm{cm}$ radius from the axis of the tube), two points on the middle partition (one at the center, the other at $0.25-\mathrm{cm}$ radius), and one on the rear partition (at $0.25-\mathrm{cm}$ radius). Velocity-per-fringe sensitivities were chosen as $0.386 \mathrm{~km} / \mathrm{s}$ (VLNV-03) and $0.846 \mathrm{~km} / \mathrm{s}$ (VLNV04). This afforded dual-delay-leg monitoring of all spots.

An additional pair of Valyn International ORB interferometers was used to monitor the motion of the X-ray input diagnostic, an aluminum disk similar to the front partition.

Data were recorded on LeCroy 7200 digitizers at $0.5 \mathrm{~ns}$ per point. Timing relative to pinch was established by assuming shock injection into the first partition at the moment of peak pinch. For the present experiment, timemarks were recorded on one digitizer and fringe records on others. Subsequent tests with timemarks injected onto all digitizers have shown that this gives 3 - $4 \mathrm{~ns}$ of jitter in the data (an amount small enough to not be a problem in the present experiment).

The overall assembly is shown in Fig. 2.2. Again, note the relatively massive holding fixtures necessary to accommodate the supporting electronics for the strain gages. The tube was softmounted to the supporting structure using large RF gaskets (essentially steel mesh over a very soft elastomer ring) so that the motion of the tube was unconstrained by the mounting system. Figure 2.2 shows the assembly as mounted adjacent to the wire arrays, which generate the Zpinch during the experiment.

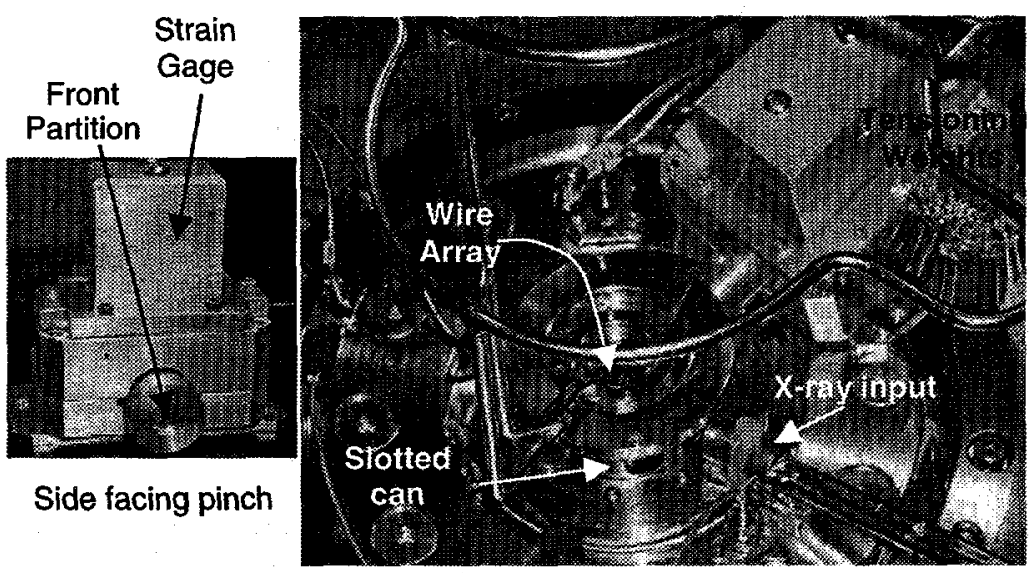

Fig. 2.2. View of tube assembly mounted to stage of Z-pinch facility. Tube in mounting is at upper right (2:30). Wires to form pinch form a cone coming upward from the center of the large circular fixture. Small weights next to tube holder assembly maintain tension on wires. Probe at lower right $(4: 00)$ is the $\mathrm{X}$-ray input diagnostic (an aluminum flyer plate similar to the front partition).

\section{NUMERICAL SIMULATIONS OF PIPE RESPONSE}

We performed a number of numerical simulations of the response of the partitioned pipe configuration, both pre-test and post-test. The calculations had three fundamental purposes. The first was to provide initial predictions of the pipe and partition responses to assist in the details of 
the experimental design. The second purpose was to help improve our understanding of the phenomenology involved in the dynamic response of the configuration in the pulsed radiation environment produced by the $\mathrm{Z}$ machine. Finally, post-shot calculations are providing validation for our modeling capabilities so that we can use them for credible and reliable predictions under other but related conditions.

For these simulations, we are using the ALEGRA code [3], which is a three-dimensional shock-physics computer program running in Lagrangian, Eulerian, and/or ALE coordinate systems. It can run on large massively parallel computers, and it has many physics options including radiation transport, $\mathrm{MHD}$, hydrodynamics, material strength (including fracture), and conduction. In the present calculations we are using Eulerian coordinates, and are employing the hydrodynamics, material strength, and radiation transport options. Although we cannot provide a detailed description here, the latter uses the $\mathrm{SP}_{1} /$ single-group approximation for the present study.

To model the partitioned pipe we used a non-uniform Eulerian mesh consisting of about 7,000 elements for the long pipe and nearly 4,000 elements for the short pipe. Two-dimensional cylindrical symmetry was used, and the pipe was surrounded by low-density gas with no opacity. The latter approach was employed to avoid using rigid boundary conditions on the pipe and to allow it to respond in a "breathing" mode if the dynamics of the problem so dictate. The axial zone sizes were large at both ends of the configuration, and were gradually reduced in dimension so that at either end of the pipe $0.2-\mathrm{mm}$ resolution was provided. In the radial direction the resolution was uniform at $1 \mathrm{~mm}$. This is not ideal, especially in terms of zonal aspect ratio, but it was the best balance between spatial resolution and computational running time. The numerical configuration is illustrated in Fig. 3.1; it is qualitatively similar for both the long and the short pipes.

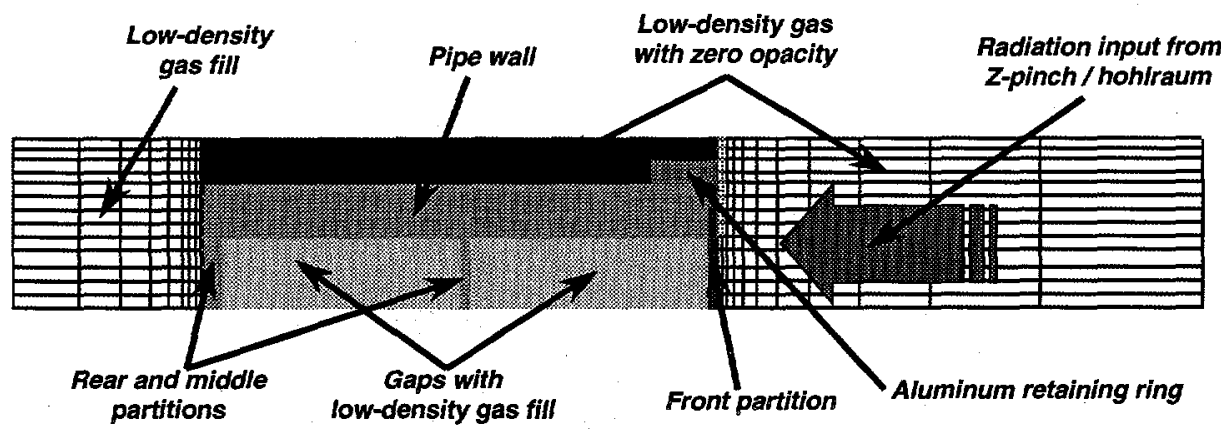

Fig. 3.1. Configuration for numerical simulations. The sketch applies to both long and short pipes, but is not proportionally scaled. For the former the gaps between the partitions are $50 \mathrm{~mm}$ and the numerical mesh involves $\sim 7,000$ elements; the latter has gaps of $25 \mathrm{~mm}$ and employs $\sim 3,800$ elements. Only the upper half of the cylindrically symmetric configuration is shown.

Both the pipe and the partitions were made of standard aluminum, and in the calculations they were modeled with the nominal Sesame equations of state. Because it is virtually all vaporized by the radiation pulse, the front partition was assumed to behave hydrodynamically, where the other two barriers and the pipe wall were treated as elastic-plastic materials with a conventional 3-kilobar yield strength. The only other materials in the problem are the low-density gases surrounding the pipe and contained in the interior. Their density was kept low to be a reasonable simulation for the vacuum that existed in the actual experiments. In addition, their opacities were set to zero so they would not significantly perturb the radiation flow.

For all of the calculations the radiation input from the Z-pinch source was characterized as a time varying Planckian or black-body temperature history. For the calculations performed before the experiments, we assumed simply that the temperature was triangular in time with a base width of $40 \mathrm{~ns}$, and a peak temperature of either 100 or $55 \mathrm{eV}$. The former represented the maximum level of loading that might be achieved in a Z-driven hohlraum situation. The lower 
temperature was our best estimate of the effective temperature anticipated at the sample location for the first experiment. The total energy fluences for these two cases differ by almost a factor of eleven $\left(\sim 200 \mathrm{~kJ} / \mathrm{cm}^{2}\right.$ for the $100-\mathrm{eV}$ peak temperature, and $\sim 19 \mathrm{~kJ} / \mathrm{cm}^{2}$ for the $55-\mathrm{eV}$ peak). They also differ in terms of their radiation spectrum, but since the front partition is optically thick at the relevant wavelengths, this is probably not a major issue.

Diagnostic measurements from the experiments yielded the actual temperature-time data shown in Fig. 3.2. Because the measurement threshold is in the region of 30 to $40 \mathrm{eV}$, the data show a baseline at this level. In the calculations we assumed that the temperature started at zero and increased to the measured value at a time of roughly $60 \mathrm{~ns}$. The curve peaks at $\sim 162 \mathrm{eV}$ and has a FWHM pulse width of $\sim 40$ ns. Due to the geometric attenuation from the slotted-can hohlraum and the $1 / \mathrm{r}^{2}$ attenuation at the actual distance from the pinch at which the pipe was located, the total energy fluence incident on the front partition was about 3.3 percent of that represented by a $163 \mathrm{keV}$ blackbody source [4]. To calculate this fluence, $\Phi$, we integrate $\sigma T^{4}(t) d t$ under the curve in Fig. 3.2, where $\sigma$ is the Stefan-Boltzman consant $\left(5.67 \times 10^{-12} \mathrm{~J} \mathrm{~cm}^{-2} \mathrm{~s}^{-}\right.$ ${ }^{1} \mathrm{~K}^{-4}$ ). By then taking 3.3 percent of the result, we estimate that the total incident fluence is $\sim 23$ $\mathrm{kJ} / \mathrm{cm}^{2}$, and it is this value that is used in the calculations.
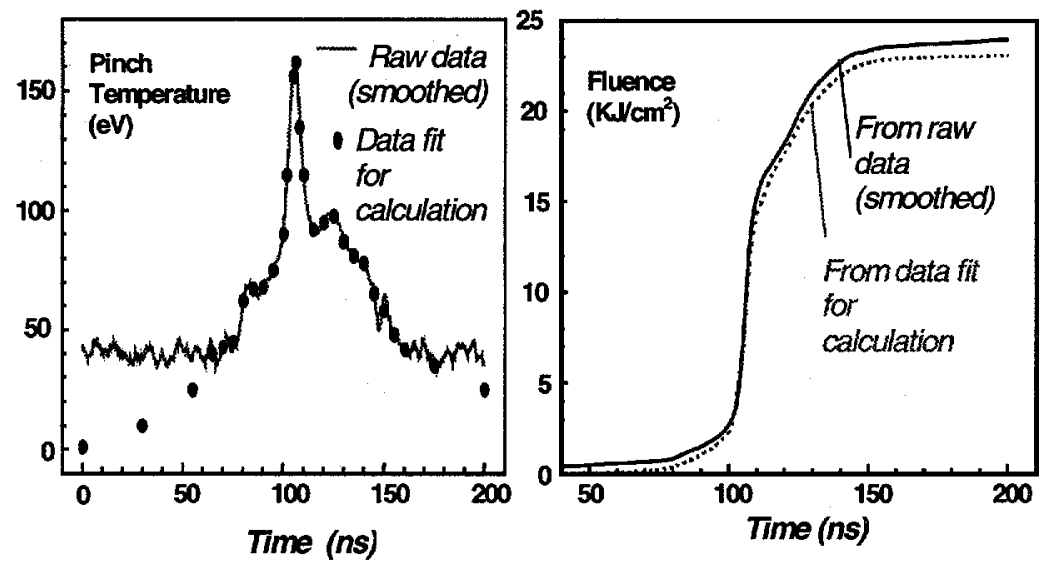

Fig. 3.2. (Left) Measured temperature/time history from the $\mathrm{Z}$ pinch for the partitioned pipe experiments. The points represent the input used for the numerical simulations. (Right) Estimate of the total energy fluence, as a function of time, incident on the front partition.

Pre-shot calculations used the simplified radiation inputs to clarify the expected responses of the pipe. We used only the long-pipe geometry, but looked at both the 100 and $55 \mathrm{eV}$ sources. The left-hand side of Fig. 3.3 shows selected plots from the two runs. Both illustrate the region from the front partition to the middle one, on the left showing the results for' a peak source temperature of $55 \mathrm{eV}$, and on the right for a peak of $100 \mathrm{eV}$. Consequently the total energy fluence is almost 11 times greater (the ratio of the fourth power of the peak temperatures) for the $100-\mathrm{eV}$ problem. The left half of each image shows the pressure, while the right half indicates the material density. In the higher fluence case $\left(T_{\max }=100 \mathrm{eV}\right.$, and $\left.\Phi=200 \mathrm{~kJ} / \mathrm{cm}^{2}\right)$, we see that the debris from the fully vaporized front partition precedes the pressure waves in the pipe wall, and by the time of the plot $(5 \mu \mathrm{s})$ it is nearly to the middle partition. In contrast, the lower fluence associated with other calculation (at $T_{\max }=55 \mathrm{eV}$, and $\Phi=19 \mathrm{~kJ} / \mathrm{cm}^{2}$ ) still vaporizes the front partition, but the resulting debris velocity is, as indicated at $10 \mu \mathrm{s}$, only about one-fourth as great. In this case the pressure waves in the pipe wall precede the debris by nearly a factor of two in time. It is this latter situation that most closely matches the anticipated experimental conditions. These two calculations clearly illustrate two different response regimes, and their dependence on total energy fluence.

Based on the experimental measurements we repeated the calculations, both for the long pipe and for the short pipe. In both cases we used the radiation input shown in Fig. 3.2. Sample results are given in the right-hand side of Fig. 3.3. As with the pre-shot calculations we show both the pressure and the density for, on the left, the long pipe, and on the right, the short pipe. The entire pipes are illustrated, including the front, middle, and rear partitions. We have picked times that show the debris just after penetrating the middle barriers. For the long pipe this is at $15 \mu \mathrm{s}$, and for the short pipe it is at $8 \mu \mathrm{s}$. These times are as expected since the physical 
dimensions are different by a factor of two. Both calculations exhibit characteristics similar to the lower temperature and fluence pre-shot problems; that is the pressure waves in the pipe walls precede the debris by about a factor of two in time.

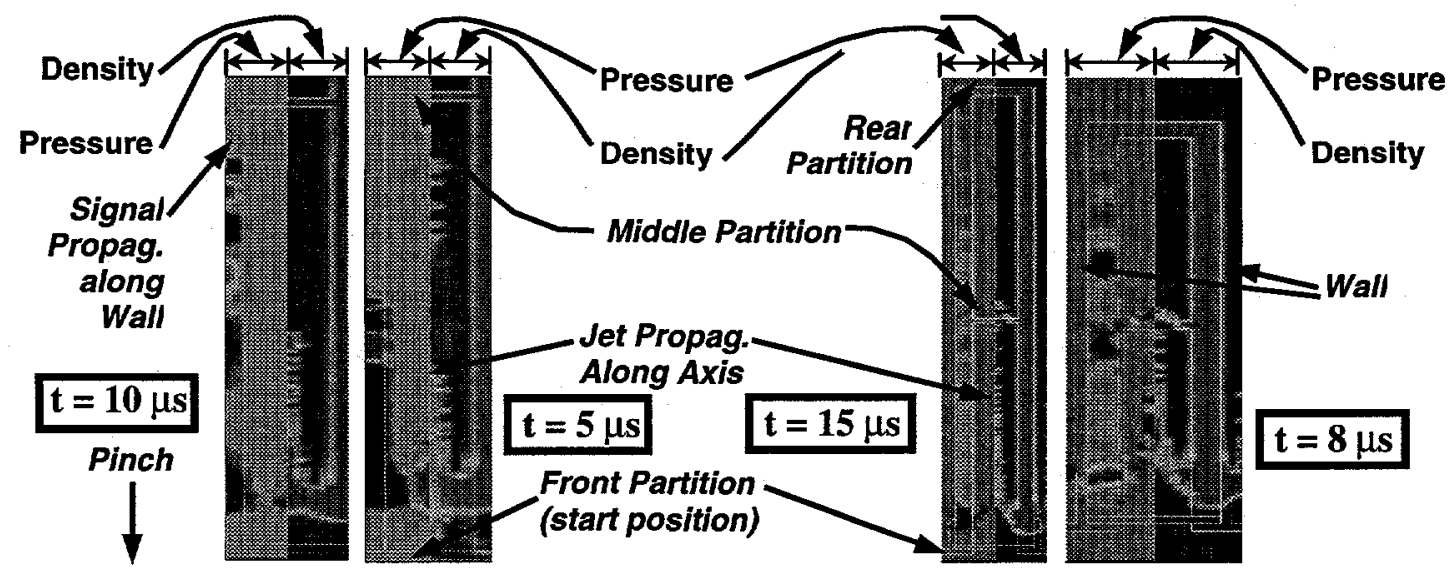

Fig. 3.3. (Left) Representative images from pre-shot calculations. The radiation inputs were 40 -nswidetriangular pulses peaking at $55 \mathrm{eV}$ (on the left) and $100 \mathrm{eV}$ (on the right). The radiation is incident from the bottom, and the total energy fluence is $\sim 11$ times greater for the higher temperature calculation on the right $\left(\sim 200 \mathrm{~kJ} / \mathrm{cm}^{2}\right.$ versus $\left.\sim 19 \mathrm{~kJ} / \mathrm{cm}^{2}\right)$. (Right) Post-shot calculations for long pipe (on the left) and short pipe (on the right). The detailed initial geometry associated with the front partition and its retaining ring can be seen at the bottom of the images. The incident fluence used in these two simulations was $\sim 23 \mathrm{~kJ} / \mathrm{cm}^{2}$.

To provide more quantitative output we plot pressure and velocity histories for Lagrangian tracer particles embedded in both the pipe wall and the three partitions, using the long-pipe calculation. Figure 3.4 shows pressure traces for four points spaced along the length of the pipe wall. The locations are, respectively, midway between the first and second partition, opposite the middle barrier, at the midpoint between the middle and rear partitions, and finally, opposite the rear partition. All these points were at the midpoint in the wall thickness. The ringing in all the traces appears to be characteristic of the loading on the front barrier. The point halfway between the first two partitions exhibits a peak pressure of almost $5 \mathrm{~kb}$, while between the second and third, the wall pressure is only a little over $1 \mathrm{~kb}$. The traces opposite the barriers are interesting because they clearly indicate the debris impacts at 13 and $35 \mu \mathrm{s}$, respectively. Both of these traces also show the initial pressure waves moving up the pipe wall, starting at times roughly half of those associated with the debris.

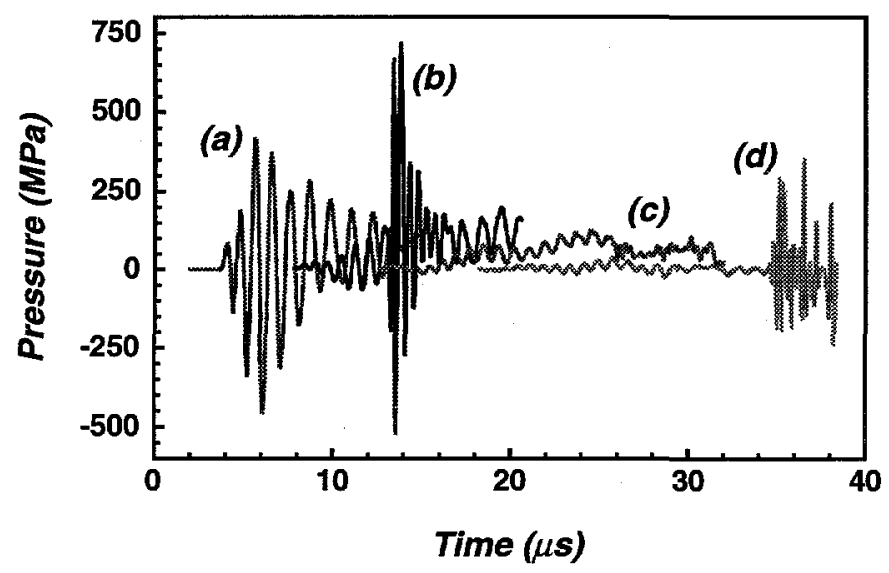

Fig. 3.4. Calculated pressure histories for points along the wall of the longpipe configuration. All points are for halfway through the wall thickness. Curve (a) is for the axial location midway between the front and middle partitions; (b) is adjacent to the middle partition; (c) is at the midpoint between the middle and rear partitions; and (d) is opposite the rear partition.

Next we plot, in Fig. 3.5, the velocity histories for the rear of each partition. For the front partition, at early times the traces show uniformity across the diameter, and peak at $\sim 4 \mathrm{~km} / \mathrm{s}$. 
The on-axis trace drops to $\sim 2.5 \mathrm{~km} / \mathrm{s}$ after penetrating the middle partition at about $13 \mu \mathrm{s}$, and drops further, to $\sim 1 \mathrm{~km} / \mathrm{s}$ or less, on impact with the rear barrier. The off-axis velocities show that after the initial interactions associated with the breakup of the front partition, the debris motion becomes strongly peaked toward the axis, especially at later times. For the middle partition, the most obvious feature is the velocity increase at $13 \mu \mathrm{s}$ from the impact of the frontbarrier debris. In an analogous fashion the velocities drop when the middle-barrier debris hits the rear partition at $34 \mu \mathrm{s}$. It is even more evident here that the debris velocities are strongly peaked toward the pipe axis. Finally, the velocities for the rear partition show the effects of both the stress waves in the pipe wall and the debris impact. The small motions from the wall signals are evident between 20 and $25 \mu \mathrm{s}$, well before the debris arrives, at $\sim 34 \mu \mathrm{s}$. The calculated rearsurface velocities of this partition are relatively low, with a maximum value a little over 0.7 $\mathrm{km} / \mathrm{s}$, but they are still strongly peaked toward the axis.
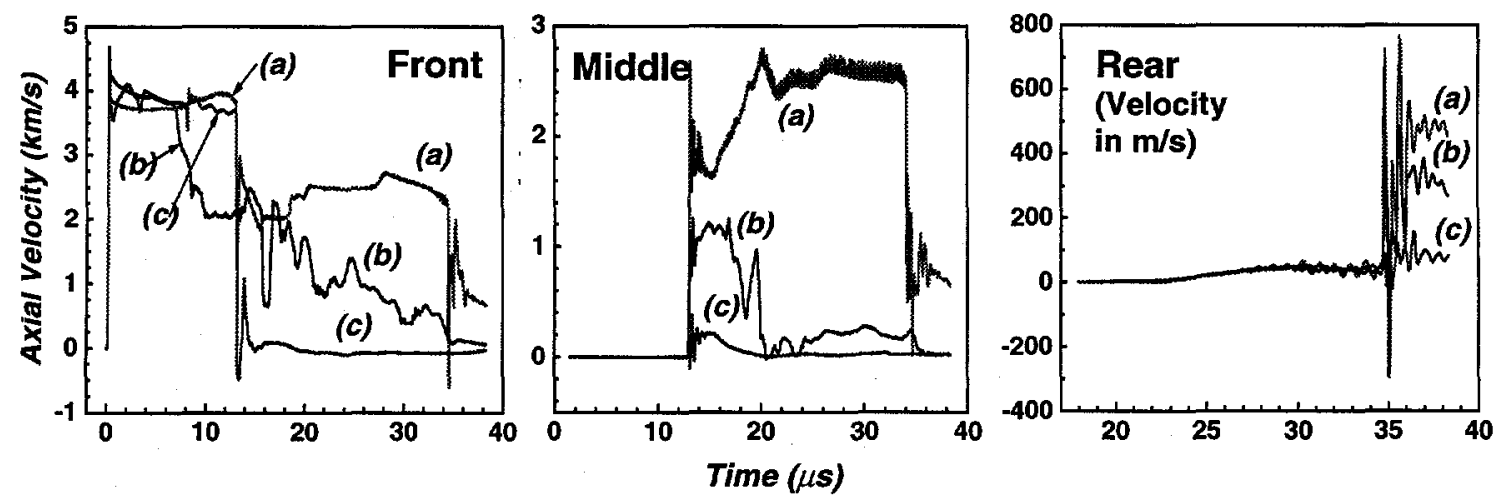

Fig. 3.5. Calculated axial velocity histories for the rear of the three partition of the long pipe. For each plot, curve (a) is for on the axis; (b) is for a radius of $0.15 \mathrm{~cm}$; and (c) is for a radius of $0.30 \mathrm{~cm}$

These calculations give particle velocities significantly higher than our measured VISAR records, but they are certainly qualitatively similar. Our best guess for the source of the discrepancy is that the total energy fluence incident on the target was probably less than we used in the calculations. It should be noted that the attenuated radiation input at the sample location was determined solely on geometric arguments. We hope that future diagnostics will give more direct results for the on-target radiation parameters, especially when complicated source configurations, like the slotted-can hohlraum, are employed.

\section{RESULTS FROM TIME-RESOLVED DIAGNOSTICS}

For the long-tube experiment, good-quality VISAR records were obtained for all channels except for one spot on the front partition; the records were unusably noisy for both VISARs for that spot. Unfortunately, the strain gages were overwhelmed by electrical noise, and so no usable data were recovered. The VISAR results are shown in Fig. 4.1.

The front partition accelerates to a velocity of about $2.2 \mathrm{~km} / \mathrm{s}$; the middle, to $0.5 \mathrm{~km} / \mathrm{s}$, and the back, to about $120 \mathrm{~m} / \mathrm{s}$. Arrival times are presented in Table 4.1 , and calculated partition velocities (based on transit times), in Table 4.2. Precursor interval velocities in Table 4.2 may be related to shock propagation along the tube walls.

On the short-tube experiment, good-quality VISAR records were obtained for all channels except for two channels on the middle partition. The VLNV-04 Data 2 record for the half-radius spot was lost when a digitizer plugin failed to trigger. As well, the VLNV-03 trace for the center spot was quite noisy, but usable. These results are shown in Fig. 4.2. The front partition accelerates to a velocity of about $1.75 \mathrm{~km} / \mathrm{s}$; the middle, to $1 \mathrm{~km} / \mathrm{s}$, and the back, to about 100 $\mathrm{m} / \mathrm{s}$. Arrival times are presented in Table 4.1, and calculated partition velocities, in Table 4.2. 

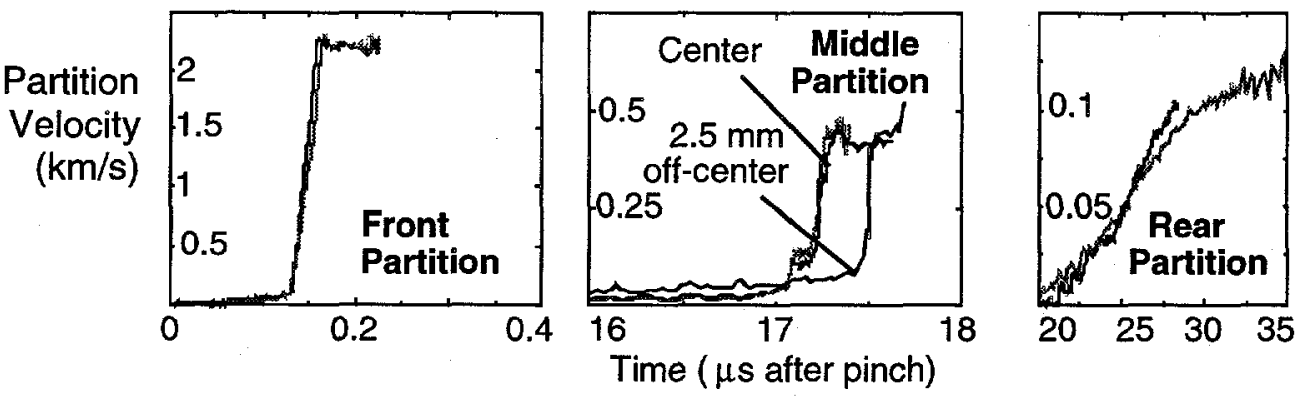

Fig. 4.1. Long-tube partition velocity histories. All times are relative to peak pinch.

Table 4.1. Observed Arrivals - Short and Long Tubes (time in $\mu$ s after peak pinch)

\begin{tabular}{|c|l|l|l|l|}
\hline & \multicolumn{2}{|c|}{ Long Tube Experiment } & \multicolumn{2}{c|}{ Short Tube Experiment } \\
\hline Partition $\downarrow$ & \multicolumn{1}{|c|}{ Toe } & Main Arrival & \multicolumn{1}{c|}{ Toe } & Main Arrival \\
\hline Front & 0.06 & 0.15 & 0.07 & 0.15 \\
\hline Middle & 9.33 edge - & 17.50 edge - & 8.2 & 9.64 edge- \\
& 12.38 center & 17.09 center & & 9.54 center \\
\hline Rear & 21.16 & $\sim 25 ?$ & 12.55 & $\sim 20 ?$ \\
\hline
\end{tabular}

Table 4.2. Precursor and Partition Velocities (calc. from observed arrival times, in $\mathrm{km} / \mathrm{s}$ )

\begin{tabular}{|l|l|l|l|l|}
\hline Interval $\downarrow$ & \multicolumn{2}{|c|}{ Long Tube Experiment } & \multicolumn{2}{c|}{ Short Tube Experiment } \\
\hline & Precursor & Partition & Precursor & Partition \\
\hline Front - Middle & 5.39 & $\begin{array}{l}2.95 \text { center } \\
2.88 \text { edge }\end{array}$ & 3.07 & $\begin{array}{l}2.66 \text { center } \\
2.63 \text { edge }\end{array}$ \\
\hline Middle - Back & 4.23 & $6.3 ?$ & 5.75 & 1.58 \\
\hline
\end{tabular}
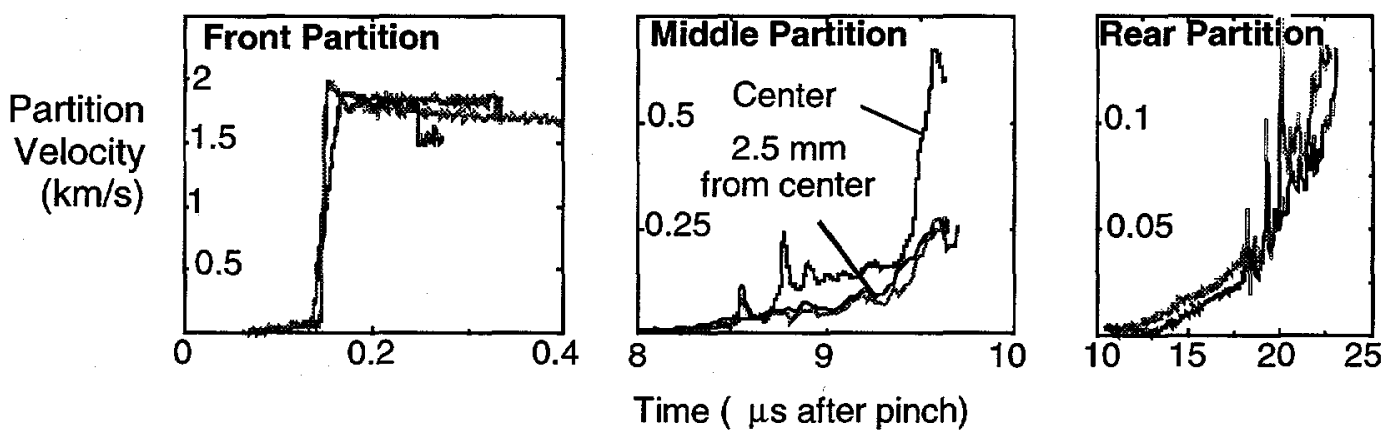

Fig. 4.2. Short-tube VISAR velocity histories. All times are relative to peak pinch.

In both experiments, the front partition was abruptly accelerated to $\sim 2 \mathrm{~km} / \mathrm{s}$. Loss of signal after a short period (less than $300 \mathrm{~ns}$ ) is consistent with (1) a loss of reflectivity of the partition due to shock breakout, and (2) an expected impact of the surface on the fiber ends at about 250 ns after the start of surface motion. An apparent decrease in surface velocity of this partition is probably an artifact, caused by the change in direction required of light traversing the path from the sending fiber to the surface, and into a receiving fiber. 
On the middle partition, the primary arrival appears to impact the center $10-40 \mathrm{~ns}$ earlier than the edge (short and long tubes, respectively). Modeling of the experiment using ALEGRA (previous section) predicts this as well, and suggests that the front partition does not travel as a disk, but as a debris pattern strongly focussed on the axis of the tube. The mean velocity of the main impacting mass along the first half of the tube length $(2.9 \mathrm{~km} / \mathrm{s}$ for the long tube and 2.6 $\mathrm{km} / \mathrm{s}$ for the short tube) is slightly greater than the values suggested by VISAR measurements of the initial launch of the front partition $(2.2 \mathrm{~km} / \mathrm{s}$ for the long tube, $1.8 \mathrm{~km} / \mathrm{s}$ for the short tube).

It is difficult to identify a clean arrival for the back partition in either experiment. This is consistent with the arrival of a dispersed cloud of debris, melt, and/or vapor, and strongly suggests that the middle plate loses integrity in the course of the experiment, as also suggested by numerical simulations.

The toes of the waveforms carry additional information. For the long-tube experiment, they suggest a signal propagating along the walls of the pipe at $\sim 5.4 \mathrm{~km} / \mathrm{s}$. This is consistent with the velocity of a low-amplitude shock propagating in aluminum. Results for the short tube experiment show more scatter.

\section{RESULTS FROM POST-EVENT ANALYSIS}

Both tubes were recovered after the respective experiments. The long tube remains integral. The short tube was sliced lengthwise by diamond saw and examined, both visually and from a metallography perspective. Figure 5.1 shows photographs of one of the two halves.

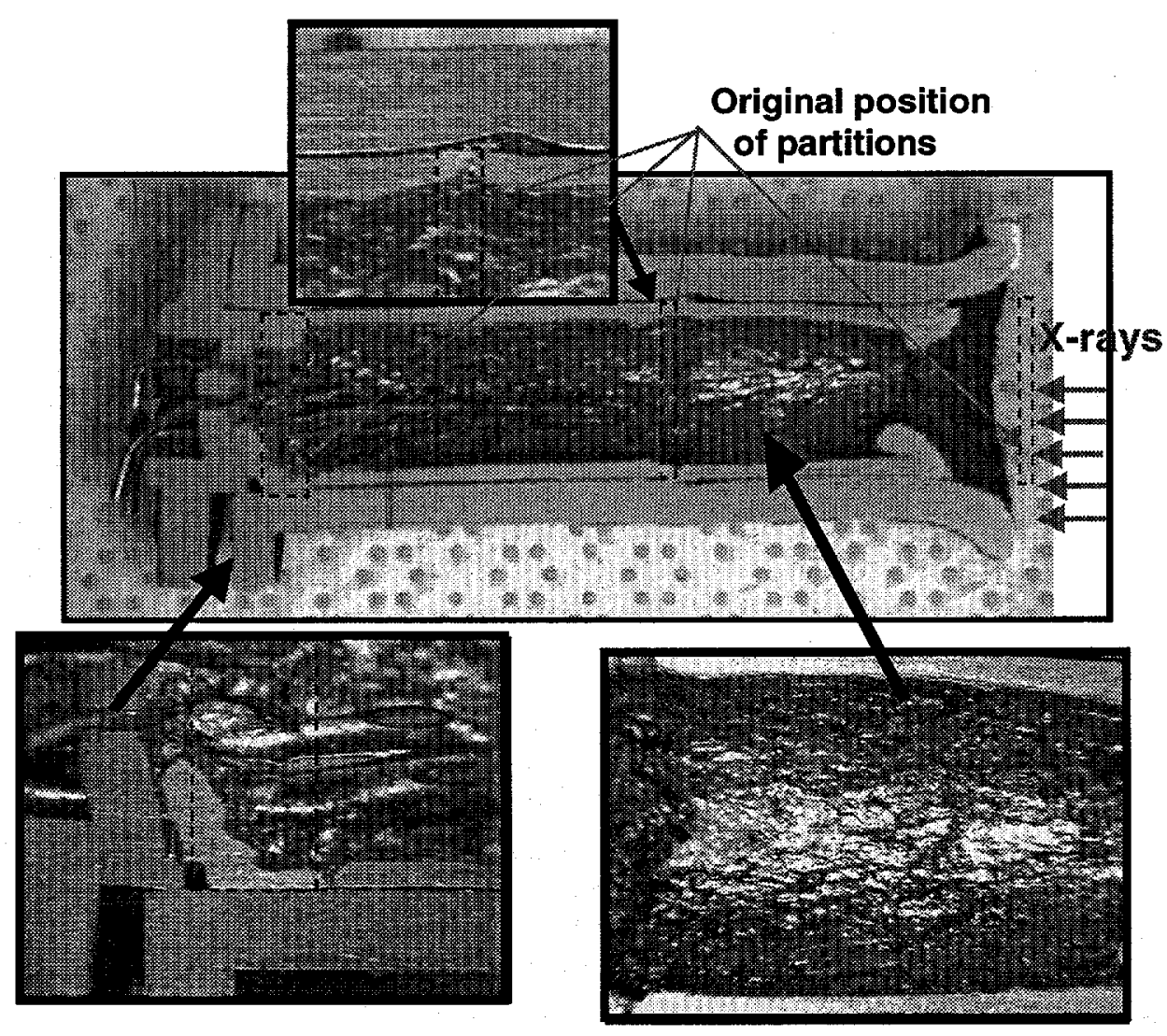

Fig. 5.1. Post-test photographs of half of the short tube. Original positions of aluminum partitions are shown by dashed rectangles (displaced downward for clarity). 
It is clear that a combination of violent processes has occurred. Specific observations are:

1) No recognizable macroscopic fragments remain from the front and middle partitions. The edge portions of the rear partition are recognizable, although the central half is destroyed.

2) Some of the original steel tubing used to support the VISAR fibers is still present, although it is discolored as if by heat.

3) There is an abundance of bubbles in this metal, occasionally to the point of a spongy texture.

4) There are numerous translucent whitish spots on the remaining lengths of steel tubing. There is an excoriated appearance to portions of the steel tubes and the walls.

5) The material at the distal end of the tube is preferentially distributed toward the walls.

6) Additional material deposited on the inside of the tube assembly throughout the $30 \%$ distal from the pinch shows vesicles consistent with partial vaporization.

7) The front of the main tube has been flared, and the front of the separator tubing inside the main tube has been folded back.

8) General structural deformation reflects large stresses transmitted through the tube. The liner is slightly separated from the outer wall of the pipe. The distal end of the tube has been noticeably strained.

Several interpretations may be advanced based on these observations:

1) The metal on the inside of the pipe is definitely cooled from a melt. This is indicated by the texture.

2) The front and middle partitions were pervasively melted, and at least the front of the front partition was vaporized.

3) Enough aluminum vapor was generated to form the majority of the bubbles.

4) Note that the barriers were nearly pure Al, while the tube structure was 6061-T6.

5) The whitish spots on the steel tubing may be oxidized aluminum [if an atmosphere was present], or may be artifacts of the process of bisecting the recovered structure.

6) The debris/vapor/liquid flow excoriated the outsides of the steel tubes and the inner wall of the pipe.

The other half of the short tube was analyzed by scanning electron microscopy and microprobe for further information about tube/partition behavior during the experiment. Representative results are shown in Fig. 5.2. "BSE" is backscattered electron imaging (utilizing low-energy electrons); this generally produced better contrast than did scanning electron microscope settings. Although numerous elemental images were taken (selecting x-ray energies characteristic of elements of interest to produce elemental maps), results from these will be summarized in text form in the present paper.

Comments about specific sites follow (see letters on Fig. 5.2 images for locations):

A) (Outside of tube on front flare) Aluminum structure has "skin" $30-50$ microns thick of turbulently mixed aluminum and stainless steel. Sample composition was $48 \% \mathrm{Al}, 23 \% \mathrm{Fe}$, balance a mixture approximately that of stainless steel.

B) (Outside of tube about $25 \%$ of the way back) 2 layers of melt solidified. The basal layer is steel-rich; the outer is aluminum-rich. Turbulence of $(A)$ is not observed.

C) (Inside tube, near base) Three distinct layers are observed above the aluminum substrate. The outer layer is coarsely vesicular, ranging to 600 - 700 microns in thickness (more locally), with exsolved intermetallic crystals of iron aluminides (with some $\mathrm{Cr}$ and $\mathrm{Ni}$ ). These are likely to be $\mathrm{Cr}$ and Ni-bearing $\mathrm{FeAl}_{3}$. The next, also iron-rich, appears to be a chill zone $\sim 150$ microns thick. Delamination is observed between these two layers. The deepest layer is $\sim 35$ - 40 microns thick, and appears to be a diffusion zone (iron into aluminum substrate).

D) (Outside of fold point on spacer tube; proximal to pinch) Note that some copper is present here, possibly from copper tape used to secure near-pinch wiring.

E) (Inside of fold on spacer tube; opposite side from (D).) Layered "coxcomb" texture. (Outside of tube about $30 \%$ of the way back; opposite side from (B).) Weight percentages 

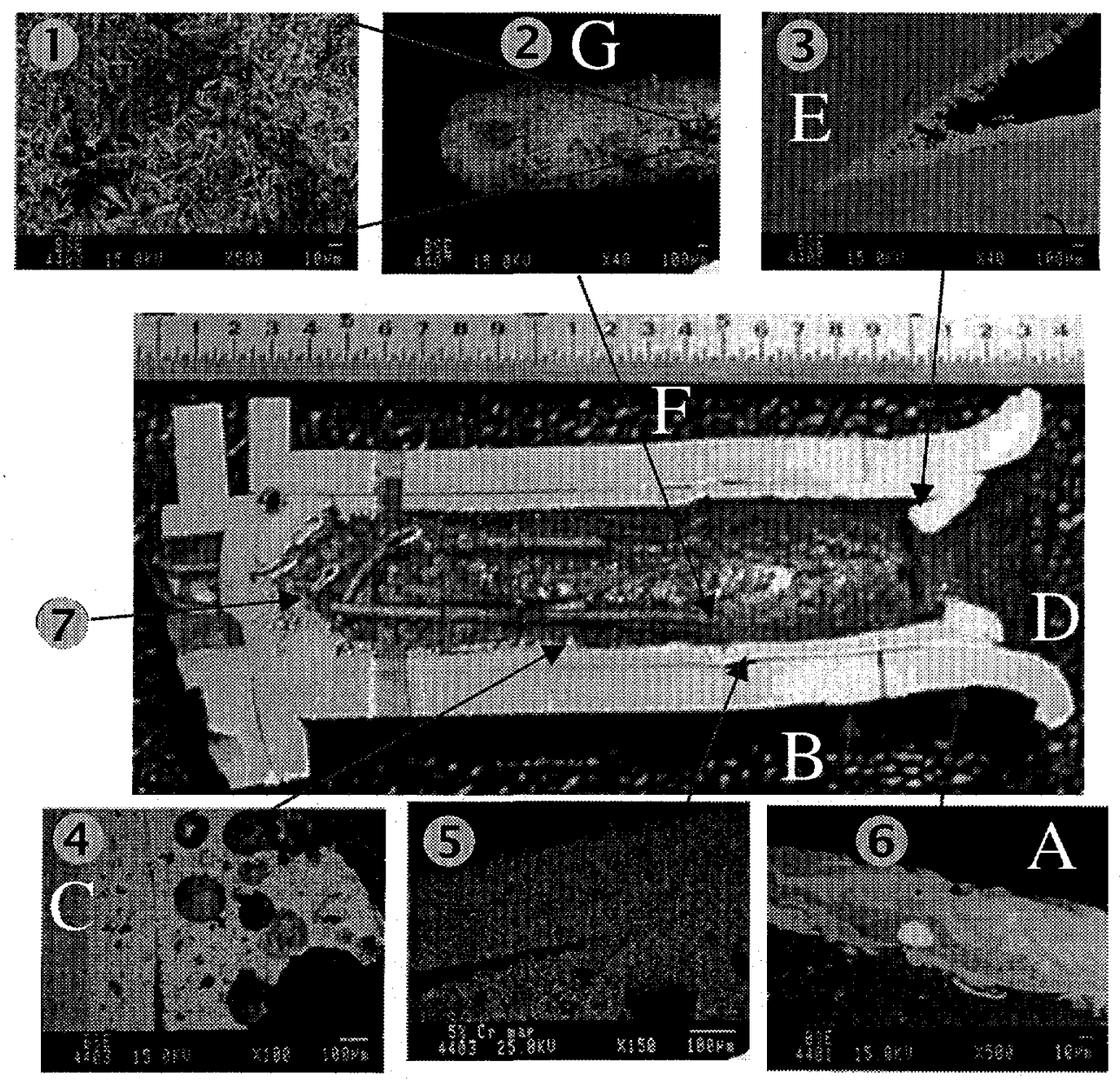

Fig. 5.2. Representative microprobe (plate (5) and SEM (BSE) results from short tube experiment. Ruler on reference photo is in inches. Scale bars at lower right corners of (1) and (6) are 10 microns; others are 100 microns. Letters are to reference locations discussed in text.

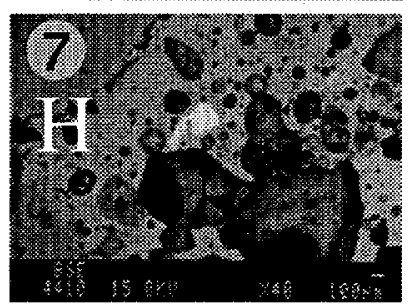

are $20 \% \mathrm{Ni}, 10-11 \% \mathrm{Cu}$, balance $\mathrm{Al}$.

F) (Eroded tip region of steel tubing from fiber optics) The white patches observed optically are alumina, and are several microns thick.

G) The region inside the tube at the distal end from the pinch contains an appreciable volume of what appears to be an coarsely vessicular aluminum matrix saturated with ferrous metals.

These observations suggest the following inferences:

1) The outside of the tube, which shows thin layers of cryptocrystalline, turbulently mixed metal compositions, cooled rapidly after the initial flux of X-ray energy.

2) The middle of the structure, which contains a more homogenized elemental mixture with a porphyritic texture (isolated crystals in a cryptocrystalline matrix), remained hotter longer than the outside.

3) Aluminum was oxidized within the structure at the steel tubes to produce aluminum oxide. The source of oxygen, or the reduced material, is a mystery inasmuch as the test was conducted under vacuum. It seems unlikely that the aluminum oxide was introduced during the later cutting process. The possibility must be considered that air remained in the tube. 
One of the potentially greatest benefits of the microprobe in this analysis, mapping the final locations of the partitions, could not be readily achieved because of the similarity between the $1100 \mathrm{Al}$ composition of the partitions (pure Al) and the 6061-T6 composition of the tubes. The composition of 6061-T6 aluminum is $98 \% \mathrm{Al}$, with $\leq 0.7 \mathrm{wt} \% \mathrm{Fe}, \sim 1 \mathrm{wt} \% \mathrm{Mg}$, and smaller amounts of $\mathrm{Si}, \mathrm{Mn}, \mathrm{Zn}, \mathrm{Ti}, \mathrm{Cu}$ and $\mathrm{Cr}$ [5]. In future tests it may be worth considering making partitions and pipe (or other vessel) of different elements to better allow such mapping.

\section{CONCLUSIONS}

In these two experiments, several diagnostics have been used to observe debris flow in a tube subject to a strong cold x-ray fluence from a Z-pinch peaking at approximately $160 \mathrm{eV}$. Both velocity interferometry and post-test (metallurgical and microprobe) analysis suggest a violent flow pattern including loss of plate integrity and preferential rapid propagation along the center channel. The post-shot analysis also suggests the extensive melting and partial vaporization of the front and middle partitions occurred. This is dramatically different from the behavior expected for normal aluminum plate impacts at similar mean velocities $(\sim 2-2.5 \mathrm{~km} / \mathrm{s})$, where melting would not be expected.

It is extremely difficult to deploy strain gages in the $Z$ environment due to the electromagnetic interference present during the experiment $\left(10^{13}-10^{14}\right.$ amps adjacent to the gages, as well as large currents in many parts of the hibay).

In future experiments, it may be worthwhile to fabricate partitions and tubes (or other elements) from different elements so that microprobe mapping of final part distributions may be more easily done.

These experiments successfully combined the unique $\mathrm{X}$-ray production aspects of the Zfacility with a much larger number of shock compression diagnostics than previously used in this environment. A worthwhile extension of this work will be to include the penetrating $x$-ray imagery expected from the Z-Backlighter diagnostic in future experiments. This will provide real-time imagery of debris flow in pipes such as described here.

Numerical simulations qualitatively match the dynamic response of the configurations, but appear to overpredict the debris velocities. We feel that an overestimate of the total energy fluence on the sample is probably the source of the discrepancy. We anticipate that diagnostics will be improved on future experiments of this type.

Acknowledgement-This work was performed at Sandia National Laboratories and supported by the U.S. Department of Energy under contract DE-AC04-94AL85000. Sandia is a multiprogram laboratory operated by Sandia Corporation, a Lockheed Martin company, for the USDOE.

\section{REFERENCES}

[1] Hall CA, Asay JR, Trott WM, Knudson M, Fleming KJ, Bernard MA, Clark BF, Hauer A and Kyrala G, Aluminum Hugoniot measurements on the Sandia Z Accelerator, in Shock Compression of Condensed Matter -1999, Furnish MD, Chhabildas LC and Hixson RS, editors, 2000, pp. 1171-1174. See also Bailey JE, Asay J, Bernard M, Carlson AL, Chandler GA, Hall CA, Hanson D, Johnston R, Lake P and Lawrence J, Optical spectroscopy measurements of shock waves driven by intense z-pinch radiation, J. Quant. Spectr. \& Radiative Transfer, 2000; 65(1-3), 31-42.

[2] (e.g.) Peterson RR., Response of National Ignition Facility first wall materials to target X-rays and debris, Fusion Technology, 1996; 30(3), 778-782.

[3] Summers RM, Peery JS, Wong MK, Hertel ES Jr., Trucano TG and Chhabildas LC, Recent progress in ALEGRA development and applications to ballistic impacts, Int. J. Impact Engng., 1997, 20, 779-788.

[4] Vesey R, personal discussions, 1999.

[5] Matweb: Available on the World Wide Web at http://www.matweb.com; see under Material Type, 6000-series aluminum alloys. Essentially similar information available from Metals Handbook Vol.2 - Properties and Selection: Nonferrous Alloys and Special-Purpose Materials, ASM International 10th Ed. 1990. 